\title{
A MODEL FOR PANDEMIC CONTROL THROUGH ISOLATION POLICY
}

\author{
Sibusiso Moyo ${ }^{1}$, Luis Gustavo Zelaya Cruz ${ }^{2}$, Rafael lima de Carvalho ${ }^{3}$, \\ Roger Marcelin Faye ${ }^{4}$, Pavel Yaroslav Tabakov ${ }^{1}$ and Felix Mora-Camino ${ }^{5, *}$
}

\begin{abstract}
In this paper we model the dynamics of a spreading pandemic over a country using a new dynamical and decentralised differential model with the main objective of studying the effect of different policies of social isolation (social distancing) over the population to control the spread of the pandemic. A probabilistic infection process with time lags is introduced in the dynamics with the main contribution being the proposed model to explicitly look at levels of interaction between towns and regions within the considered country. We believe the strategies and findings here will help practitioners, planners and Governments to put in place better strategies to control the spread of pandemics, thus saving lives and minimizing the impact of pandemia on socio-economic development and the populations livelihood.
\end{abstract}

Mathematics Subject Classification. 34A34, 49K30, 37M05.

Received July 19, 2020. Accepted November 18, 2020.

\section{INTRODUCTION}

In this study the modelling of dynamics of a spreading pandemic over a country is considered. A new dynamical and decentralised differential model is proposed with the objective to study the effect of different politics of social isolation over the population to control the spreading and inforce the containment of the pandemic. Beside the introduction of a probabilistic infection process with time lags in the dynamics of pandemia, the main contribution of the proposed model is to introduce explicitly the level of interaction between people of towns or regions composing the considered country. This allows to characterize fully isolation plans at the regional level and to formulate optimal control problems. Two optimal control problems are formulated with the aim of either minimizing the death toll or to restart economy as soon as possible. These two objectives have been already the subject of polemics in different countries during the present CV19 pandemic. Numerical simulations are produced to display the behaviour of the pandemic under different isolation policies with a view of understanding the dynamics and how best to inform planning strategies to manage the spread and combat the pandemic.

Keywords. Pandemic modelling, spatial dynamics, optimization, simulation.

1 DUT, South Africa.

2 UFF, Brazil.

3 UFT, Brazil.

4 UAM, Senegal.

5 DUT/UFF/ENAC, France.

${ }^{*}$ Corresponding author: felixmora@id.uff.br 
The paper is organised as follows: Section 2 proposes a brief overview of epidemic and pandemic modelling, Section 3 introduces the DUT-F model, Section 4 presents an analysis of the dynamics of the DUT-F model, Section 5 displays preliminar simulation results, Section 6 introduces a state space representation of the model, then through optimal control theory, Section 7 considers the minimization of the duration of the pandemia and Section 8 considers the minimization of the death toll during the pandemia, and finally in Section 9, the conclusion presents a review of the obtained results as well as ideas for further research on the subject.

\section{Modelling of Disease Propagation}

A pandemic [15] is a disease affecting persons over a wide geographical area while an epidemic concerns the appearance of a particular disease in very large number of people during a given period of time. Then the notion of pandemic covers a spatial dimension which is not in general fully considered when tackling with epidemics. However, the majority of mathematical models developed in the field of disease propagation [5] are of the epidemic type. The objective of their construction is at first to understand the underlying epidemic mechanism and to formalize it through mathematical equations (differential equations in general). The calibration of their parameters which should lead to the proper validation of these models is often approximate. This limitation arises from the poor quality of the available data which are frequently incomplete and validation is often performed at a qualitative level by comparison with broad observation. Then many of these quantitative models are in fine used to develop, through the qualitative analysis of their behaviour (existence of equilibrium states at the endemic level, asymptotic convergence or divergence from attractors, existence of threshold levels for the epidemic to start or die for good, etc) general control strategies (vaccination, treatment, quarantine, etc). In recent decades and years, models have been developed to analyse HIV/AIDS [4,11], H1N1 [2], SARS [13] and very recently COVID19 dynamics.

In this field as in other applications fields of mathematical modelling one important dilemma is to choose an adequate level of details: a simplified model is easy to build but quite useless to help to quantify effective control policies while complex theoretical models are difficult to validate and then have no practical outcome Complex microscopic models have been developed to model the infectious process at the individual level [1] using advanced graph theory stochastic processes and cellular automata However when treating the case of a whole population their associated computational burden turn them inoperent and only macroscopic models are considered The more classical macroscopic model of epidemic modelling is the SIR model [8] where Susceptible Infectious and Recovered stocks of people interact according to nonlinear dynamics such as:

$$
\dot{S}=-\beta \cdot S \cdot I, \dot{I}=\beta \cdot S \cdot I-k \cdot I \quad \text { and } \quad \dot{R}=k \cdot I
$$

where $\beta$ and $k$ are positive parameters.

Different attempts have been performed in the past to provide a spatial dimension to this type of model. For example in [7] a model has been proposed to describe the spread of influenza in Europe through air travel where susceptible, contagious but asymptomatic and recovered people are cleared for air travel and where spreading is modelized considering the arriving or departing number of contagious travelers which modify the health status composition at each air connected town which follows their local SIR model. In [16], a discrete time decentralized version of the SIR model has been used to define effective regional vaccination plans through optimal control theory where vaccinated people go from susceptible to recovered local stocks, while no isolation parameter is introduced in the model. In the last few months, SIR-type models have been proposed to describe, predict or reproduce the spreading of the on-going COVID19 epidemic in different countries:

- In [3], the classical global SIR model has been modified to stuck to the currently believed spreading behaviour of this disease by introducing new categories (compartments) of people with respect to COVID19: exposed, asymptomatic, symptomatic and dead. This study which has been illustrated by the case of South Africa considers no explicit isolation parameter allowing to temper the development of the disease. Other recent similar works are $[10,12]$. 
- In reference [14], a multicity modelling of COVID19 epidemic considers explicitly the spatial dimension of the pandemia, in this study applied to the case of India the proposed epidemic interaction process between towns turns difficult the satisfaction of the overall constant population assumption.

\section{The DUT-F MODEL}

The DUT-F (DUT-UAM-UFT-UFF) model proposes a new approach to the modelling of the development and spreading of a pandemia across a country composed of different centers. This model is developed by the Durban University of Technology in South Africa in cooperation with the Amadou Mahtar Mbow University of Senegal and the Federal Universities of Tocantins and Fluminense of Brazil.

In this model, a country is represented by a set of $n_{T}$ interconnected towns representing the different regions whose populations are $P_{i}, i=1$ to $n_{T}$. It is supposed that the population of a town is equally attained by the infection independently of age, gender or social status, but different sanitary conditions can be present between towns. Let define for town $i$ at time $t$ the different stocks of people considered in the study: $E_{i}(t)$ : the number of exposed people, in general not directly known; $A_{i}(t)$ : the number of asymptomatic infected people, in general not directly known; $S_{i}(t)$ : the symptomatic infected people, supposed known; $N_{i}(t)$ the amount of naturally recovered people, in general not directly known; $C_{i}(t)$ the amount of cured symptomatic people, supposed known; $D_{i}(t)$ the number of dead people, supposed known; $T_{i}(t)$ the number of candidates to testing.

Assumptions about the infection process are the following: for simplicity the rates of natural births and deaths other than from pandemic, are not considered during the period of study and if considered could be considered equal leading again to the constant population assumption. Also, imigration/emigration flows between towns are supposed negligeable during the considered time period while short term two ways interactions are considered. All sane people, except the recovered from infection, are exposed to infection. The probability of infection in an elementary interaction between a sane person of city $i$ and an asymptomatic infected one of city $j$ is $\beta_{i j}$. Asymptomatic infected people recover naturally at a rate $\rho_{A N i}$ after a recovery time $\tau_{N i}$. Asymptomatic infected people become symptomatic at a rate $\rho_{A S i}$ after an incubation time $\tau_{S i}$. All symptomatic people are supposed immediately removed from public space and receive treatment in an isolated place, hospital or other. Alternatively it could be assumed that there is a residual infection process from symptomatic people due to isolation flaws during medical treatment. No infection of health professional treating patients is considered in this study. A rate $\rho_{S C i}$ of symptomatic people recovers through treatment after a treatment time $\tau_{T i}$, and it is further assumed that the symptomatic people are no longer exposed to the pandemia. Symptomatic people who die at a rate $\rho_{S D i}$ after a treatment time $\tau_{D i}$.

Population interaction levels inside town and between towns (Fig. 1) are given by origin-destination symmetric matrices where the interactions are assumed to be proportional to round trips per unit of time: $\left[T_{i j}^{\mathrm{min}}\right]$ is the $n_{T} \times n_{T}$ matrix representing the interaction level associated to essential services; intermediary levels of interactions could be adopted where $\left[T_{i j}^{k}\right]$ is a $n_{T} \times n_{T}$ matrix representing a $k$ th interaction level associated to partially reduced services; $\left[T_{i j}^{n}\right]$ is the $n_{T} \times n_{T}$ matrix representing the interaction level associated to nominal services. These matrices can be computed from data about accessibility produced by urban and regional planning organizations. When there is no noticeable direct interaction between town $i$ and $j, T_{i j}=T_{j i}=0$.

According to the adopted assumptions about the pandemic, the different stocks of people are related by a succession of flows as represented in Figure 2:

With the adopted assumptions, the number of recovered people is $R_{i}(t)=N_{i}(t)+C_{i}(t)$, the number of people interacting in the infection process is $E_{i}(t)+A_{i}(t)+R_{i}(t)$, the number of infected people is $I_{i}(t)=A_{i}(t)+S_{i}(t)$, the number of sane people is $E_{i}(t)+R_{i}(t)$, the number of people candidate to testing is $T_{i}(t)=E_{i}(t)+A_{i}(t)+$ $N_{i}(t)$ and the population composition for town $i$ at time $t$ is given by:

$$
P_{i}=E_{i}(t)+A_{i}(t)+S_{i}(t)+N_{i}(t)+C_{i}(t)+D_{i}(t) i=1 \quad \text { to } n_{T} .
$$




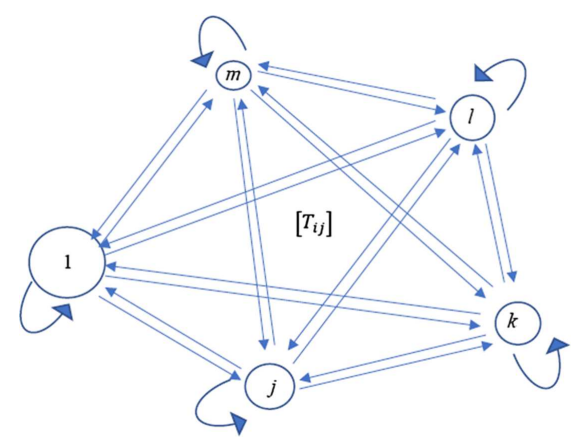

FIGURE 1. Interactions within the regional/urban system.

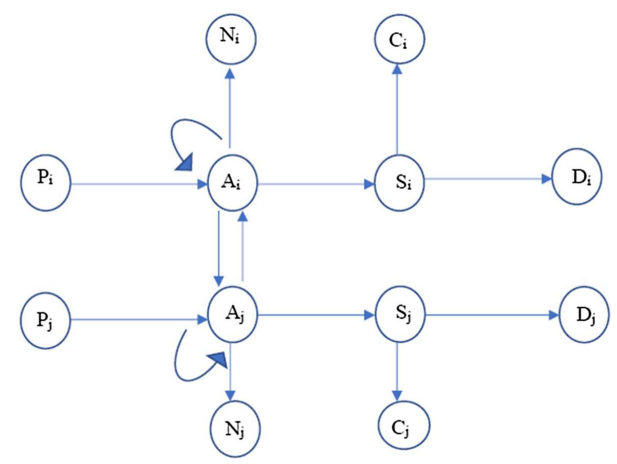

Figure 2. Flow chart of pandemia interactions.

Although the proposed model is a time continuous one, the unit of time considered in this study is the day. The mean rate of infection at town $i$ is given by:

$$
\begin{aligned}
\dot{A}_{i}(t)= & \beta_{i i} T_{i i}(t) \cdot \pi_{i i}(t)+\sum_{j=1, j \neq i}^{n_{T}} \beta_{j i} T_{j i}(t) \cdot \pi_{i j}(t) \\
& +\sum_{j=1, j \neq i}^{n_{T}} \beta_{j i} T_{i j}(t) \cdot \pi_{i j}(t)-\rho_{A N i} \cdot A_{i}\left(t-\tau_{N i}\right)-\rho_{A S i} \cdot A_{i}\left(t-\tau_{S i}\right)
\end{aligned}
$$

where

$$
\pi_{i j}(t)=\left(\frac{E_{i}(t)}{\left(E_{i}(t)+A_{i}(t)+R_{i}(t)\right)}\right) \cdot\left(\frac{A_{j}(t)}{\left(E_{j}(t)+A_{j}(t)+R_{j}(t)\right)}\right) .
$$

Here $\pi_{i i}(t)$ is the probability that an interaction is between a sane infectable person of town $i$ and an infected one of the same town, $\pi_{i j}(t)$ is the probability that an interaction between a person of town $j$ coming at town $i$ is between an exposed person of town $i$ and an asymptotic person of town $j$. The third term of the RHS of (3.2) corresponds to an exposed person from town $i$ who gets infected in town $j$ and comes back to town $i$. Then the rate of symptomatic people is given by:

$$
\dot{S}_{i}(t)=\rho_{A S i} \cdot A_{i}\left(t-\tau_{S i}\right)-\rho_{S C i} \cdot S_{i}\left(t-\tau_{T i}\right)-\rho_{S D i} \cdot S_{i}\left(t-\tau_{D i}\right) .
$$

The rate of natural recovery of asymptomatic people is given by:

$$
\dot{N}_{i}(t)=\rho_{A N i} \cdot A_{i}\left(t-\tau_{N i}\right) .
$$


The rate of treated recovery of symptomatic people is given by:

$$
\dot{C}_{i}(t)=\rho_{S C i} \cdot S_{i}\left(t-\tau_{T i}\right)
$$

The rate of death of symptomatic people is given by:

$$
\dot{D}_{i}(t)=\rho_{S D i} \cdot S_{i}\left(t-\tau_{D i}\right) .
$$

The number of exposed people at town $i$ is:

$$
E_{i}(t)=P_{i}-\left(A_{i}(t)+S_{i}(t)+N_{i}(t)+C_{i}(t)+D_{i}(t)\right)
$$

with

$$
0 \leq E_{i}(t) \leq E_{i}(0)<P_{i} \quad i=1, \ldots, n_{T} .
$$

Observe that relations (3.7) and (3.8) imply that the domains of variation of the different stocks are bounded and included in $\left[0, P_{i}\right], i=1$ to $n_{T}$. Here all the transmission rates $\rho_{A N i}, \rho_{A S i}, \rho_{S C i}$ and $\rho_{S D i}$ have small positive values. In the above formulation of the spatial spreading disease model, pure delays have been introduced for brievity, however other representations of delays can be more realistic. In Section 6 a state space representation is adopted where delays are approximated by first order dynamics, allowing the direct application of the Minimum Principle with the optimal control problems formulated to identify optimal isolation policies. Other approaches considering the effective distribution of delays relay on currently unavailable statistics.

\section{Analysis of the DUT-F Dynamics}

The dynamics given by equations (3.2)-(3.8) are nonlinear with time lags, so the analytic analysis of these dynamics is not straightforward. Here the initial and the final conditions are analyzed using first order approximations.

Initial behaviour: the activating event at $t=0$ generates a number of asymptomatic people $A_{i}(0)$ at town $i$, $i=1$ to $n_{T}$. Then until a time no greater than $\min \left\{\tau_{N i}, \tau_{S i}\right\}$, the asymptomatic population grows according to equation:

$$
\dot{A}_{i}(t)=\beta_{i i} T_{i i}(t) \cdot \pi_{i i}(t)+\sum_{j=1, j \neq i}^{n_{T}} \beta_{j i} T_{j i}(t) \cdot \pi_{i j}(t)+\sum_{j=1, j \neq i}^{n_{T}} \beta_{j i} T_{i j}(t) \cdot \pi_{i j}(t)
$$

until either some people recover naturally $\left(\tau_{N i}<\tau_{S i}\right)$ or some people become symptomatic $\left(\tau_{N i} \geq \tau_{S i}\right)$, this gives time to the pandemia to spread freely among exposed people. Let $\tau_{\text {start }}^{i}=\min \left\{\tau_{N i}, \tau_{S i}\right\}$, and if it is supposed that center $i$ remains isolated during the initial period $\left[0, \tau_{\text {start }}^{i}\right]$, considering that $A_{i}(t)$ remains negligeable in front of $E_{i}(t)$ which remains close to $P_{i}$ and that $T_{i i}$ remains constant, then the dynamics of $A_{i}(t)$ can be approximated by:

$$
\dot{X}(t)=\left(\beta_{i i} T_{i i} / P_{i}\right) \cdot X_{i}(t)
$$

whose solution is:

$$
X_{i}(t)=A_{i}(0) \cdot e^{\alpha_{i} \cdot t} \quad \text { with } \quad \alpha_{i}=\left(\beta_{i i} T_{i i} / P_{i}\right)
$$

which depicts an exponential growth over the initial period. Interactions between the different towns should amplify this exponential behaviour through additional contaminated sources. After time $\tau_{\text {start }}^{i}$ a part the stock of asymptotic people $A_{i}(t)$ is passed to the stock of naturally recovered people $N_{i}(t)$ or to the symptomatic people stock $S_{i}(t)$ in proportional bases w.r.t. to $A_{i}(t)$ (end of RHS of relation 2). 
TABLE 1. Some asymptotic equilibrium states.

\begin{tabular}{llllll}
\hline \hline Case & $E_{i}\left(t_{\infty}\right)$ & $A_{i}\left(t_{\infty}\right)$ & $S_{i}\left(t_{\infty}\right)$ & $R_{i}\left(t_{\infty}\right)$ & $D_{i}\left(t_{\infty}\right)$ \\
\hline Case 1 & $0<E_{o}<R_{i}$ & 0 & 0 & $0<N_{i}<P_{P i}$ & $0<D_{i}<P_{i}$ \\
Case 2 & 0 & $P_{i}$ & 0 & 0 & 0 \\
Case 3 & 0 & 0 & 0 & $P_{i}$ & 0 \\
Case 4 & 0 & 0 & 0 & 0 & $P_{i}$ \\
Case 5 & 0 & 0 & 0 & $P_{i}$ & 0 \\
\hline
\end{tabular}

When considering possible equilibrium situations, from equations (3.1) to (3.7), it appears that the system cannot anymore evolve when we have the situations represented in Table 1 where:

Case 1: $\rho_{A N i}>0, \rho_{A S i}>0, \rho_{S C i}>0$ and $\rho_{S D i}>0$. Case 2: $\rho_{A N i}=0, \rho_{A S i}=0, \rho_{S C i} \geq 0$ and $\rho_{S D i} \geq 0$.

Case 3: $\rho_{A N i}>0, \rho_{A S i}=0, \rho_{S C i} \geq 0$ and $\rho_{S D i} \geq 0$. Case $4: \rho_{A N i}=0, \rho_{A S i}>0, \rho_{S C i}=0$ and $\rho_{S D i}>0$.

Case 5: $\rho_{A N i}=0, \rho_{A S i}>0, \rho_{S C i}>0$ and $\rho_{S D i}=0$.

The general case is when all the transmission rates are strictly positive, in that case, the only possible equilibrium is when for each town, $A_{i}(t)$ and $S_{i}(t)$ go to zero while $E_{i}\left(t_{\infty}\right), R_{i}\left(t_{\infty}\right)$ and $D_{i}\left(t_{\infty}\right)$ stabilize. Then, with all the transmission rates strictly positive, assuming that the $A_{i}(t)$ and $S_{i}(t)$ are already small while the $E_{i}(t)$ and $R_{i}(t)$ change slowly, the rate of change of the $A_{i}(t)$ are majored by the following dynamics:

$$
\begin{aligned}
\dot{A}_{i}(t)= & \beta_{i i} T_{i i}(t) \cdot\left(\frac{E_{i}(t)}{\left(E_{i}(t)+R_{i}(t)\right)}\right) \cdot\left(\frac{A_{i}(t)}{\left(E_{i}(t)+R_{i}(t)\right)}\right) \\
& +\sum_{j=1, j \neq i}^{n_{T}} \beta_{j i} T_{j i}(t) \cdot\left(\frac{E_{i}(t)}{\left(E_{i}(t)+R_{i}(t)\right)}\right) \cdot\left(\frac{A_{j}(t)}{\left(E_{i}(t)+R_{i}(t)\right)}\right) \quad i=1 \text { to } n_{T} \\
& +\sum_{j=1, j \neq i}^{n_{T}} \beta_{j i} T_{i j}(t) \cdot\left(\frac{E_{i}(t)}{\left(E_{i}(t)+R_{i}(t)\right)}\right) \cdot\left(\frac{A_{j}(t)}{\left(E_{i}(t)+R_{i}(t)\right)}\right) \\
& -\rho_{A N i} \cdot A_{i}\left(t-\tau_{N i}\right)-\rho_{A S i} \cdot A_{i}\left(t-\tau_{S i}\right)
\end{aligned}
$$

Approximating the effects of time lags by:

$$
A_{i}\left(t-\tau_{i}\right) \approx A_{i}(t)-\dot{A}_{i}(t) \cdot \tau_{i}
$$

equation (4.4) becomes:

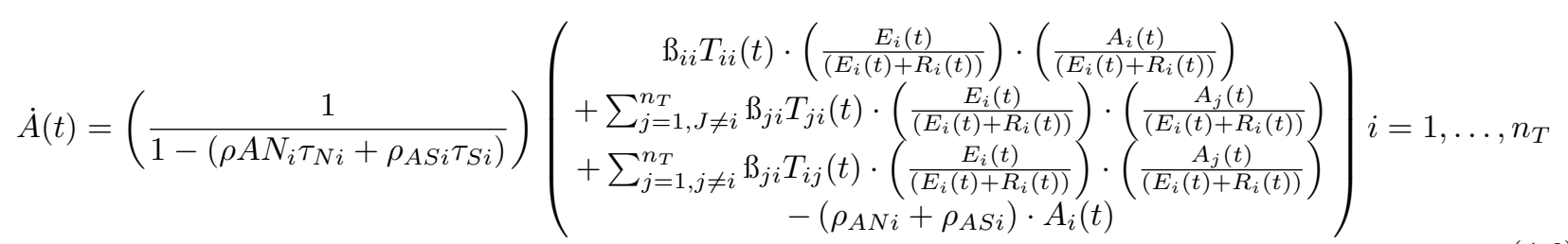

or globally:

$$
\dot{\boldsymbol{A}}(t)=\boldsymbol{M}(t) \cdot \boldsymbol{A}(t)
$$

where the rows of the $n_{T} \times n_{T}$ matrix $\boldsymbol{M}(t)$ are extracted from relation (4.7). Then a sufficient condition for asymptotic stability of (4.5) and then for (3.2) is that the eigenvalues of $\boldsymbol{M}(t)$ remain strictly negative. When 


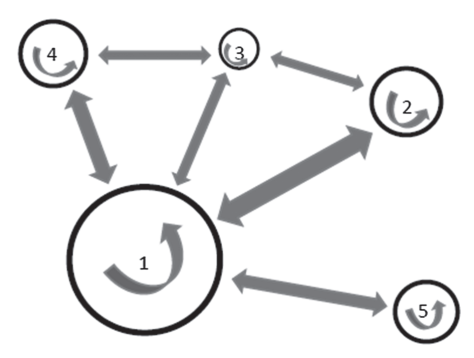

Figure 3. The considered region.

TABLE 2. Towns population and asymptomatic.

\begin{tabular}{llllll}
\hline \hline Town $i$ & 1 & 2 & 3 & 4 & 5 \\
\hline$P_{i}$ & 2000000 & 300000 & 50000 & 200000 & 150000 \\
$A_{i}(0)$ & 12 & 0 & 0 & 5 & 0 \\
\hline
\end{tabular}

TABLE 3. Normal $\left[T_{i j}^{n}\right]$ and essential $\left[T_{i j}^{\min }\right]$ levels of interaction.

\begin{tabular}{llllll}
\hline \hline Towns $i / j$ & 1 & 2 & 3 & 4 & 5 \\
\hline 1 & $4 \times 10^{12}$ & $9 \times 10^{9}$ & $25 \times 10^{7}$ & $4 \times 10^{9}$ & $225 \times 10^{7}$ \\
& $4 \times 10^{11}$ & $9 \times 10^{8}$ & $25 \times 10^{6}$ & $4 \times 10^{8}$ & $225 \times 10^{6}$ \\
\hline 2 & $9 \times 10^{9}$ & $9 \times 10^{10}$ & $25 \times 10^{6}$ & 0 & 0 \\
& $9 \times 10^{8}$ & $9 \times 10^{9}$ & $25 \times 10^{5}$ & 0 & 0 \\
\hline 3 & $25 \times 10^{7}$ & $25 \times 10^{6}$ & $25 \times 10^{8}$ & $4 \times 10^{8}$ & 0 \\
& $25 \times 10^{6}$ & $25 \times 10^{5}$ & $25 \times 10^{7}$ & $4 \times 10^{7}$ & 0 \\
\hline 4 & $4 \times 10^{9}$ & 0 & $4 \times 10^{8}$ & $4 \times 10^{10}$ & 0 \\
& $4 \times 10^{8}$ & 0 & $4 \times 10^{7}$ & $4 \times 10^{9}$ & 0 \\
\hline 5 & $225 \times 10^{7}$ & 0 & 0 & 0 & $225 \times 10^{8}$ \\
& $225 \times 10^{6}$ & 0 & 0 & 0 & $225 \times 10^{7}$ \\
\hline
\end{tabular}

considering a single center where $E_{i}(t)$ and $R_{i}(t)$ remain practically constant, the above equations reduce to:

$$
\dot{A}_{i}(t)=\frac{\beta_{i i} T_{i i}}{1-\left(\rho_{A N i} \tau_{N i}+\rho_{A S i} \tau_{S i}\right)} \cdot\left(\frac{E_{i}}{\left(E_{i}+R_{i}\right)^{2}}-\left(\rho_{R i}+\rho_{S i}\right)\right) \cdot A_{i}(t)
$$

and a sufficient condition of asymptotic stability towards 0 is reached when:

$$
\frac{E_{i}(t)}{\left(E_{i}(t)+R_{i}(t)\right)^{2}}<\left(\rho_{R i}+\rho_{S i}\right) .
$$

\section{Numerical SimUlation ILlUStration}

Here is considered a region with $n_{T}=5$ cities interconnected as shown in Figure 3 where a pandemics develops according to the proposed model. Table 2 provides the values of the population for each city where city 1 is a main urban center. Also, an example of initial infection is given by the asymptomatic people size for each town. Table 3 gives the normal (upper line) and essential (lower line) interactions levels between towns. The number 

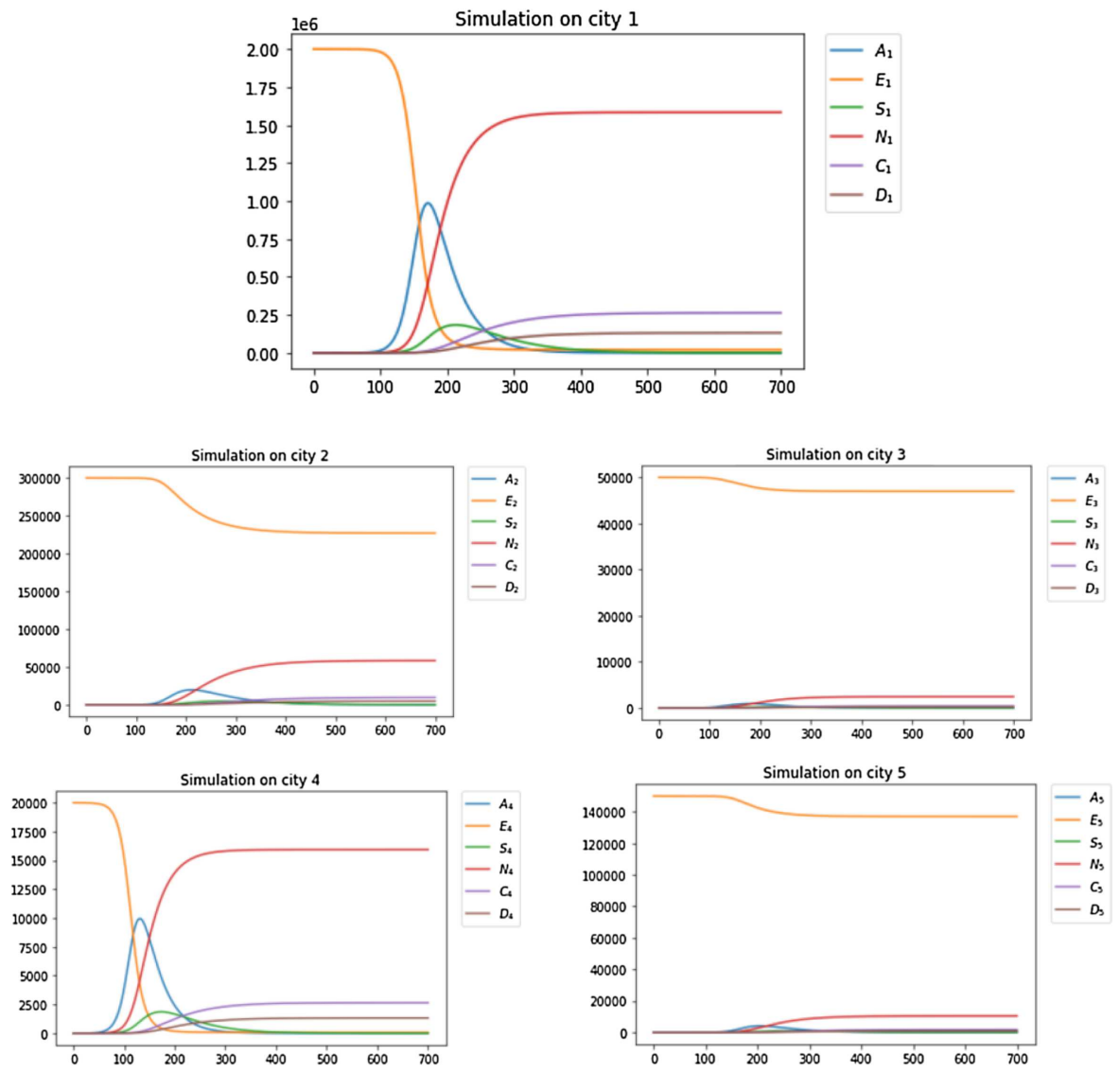

FIGURE 4. Example of evolution of the pendemic without any social isolation policy.

of daily interactions inside a city are supposed to be proportional to the square of its population, the number of interactions between two cities are supposed to be proportional to the square of the population of the less populated of the two and to be ten times smaller than its inner interactions number. It is also assumed that the number of interactions associated with essential services are ten times smaller than in the nominal situation.

In this first numerical example, it is supposed that the parameters of the pandemia are independent of the towns they apply. The values of the elementar probabilities of infection are $\beta_{i j}=4.35 \times 10^{-8} i=1, \ldots, 5$ $j=1, \ldots, 5$. The rates of transfer $\left(\right.$ in day $^{-1}$ ) from a health condition to another are: $\rho_{A N i}=0.04, \rho_{A S i}=0.02$, $\rho_{S C i}=0.027, \rho_{S D i}=0.0025 i=1, \ldots, 5$. The time lags (in days) for transfer from a health condition to another: $\tau_{N i}=6, \tau_{S i}=5, \tau_{T i}=10, \tau_{D i}=5$. These values have not been calibrated with real data from a specific pandemic but have been chosen according to a succession of hypothesis which lead to an acceptable global behaviour for the pandemic. The objective of this first scenario is to validate qualitatively the dynamics of the interdependant flows involved in the pandemic process. Figure 4 represents the evolution of the pandemic over the considered five centers from the initialization given in Table 2 during the whole pandemic in the case where no isolation policy is applied. The initial infection can be either the result of the importation of the virus or of an endogenous generation of it. The time scale (abscissa) is given in days in the different following graphical simulation results.

It appears that the early beginning of the infection is very slow and it could take about three months to become a noticed health issue for authorities. Then the infection process knows a strong acceleration to attain 

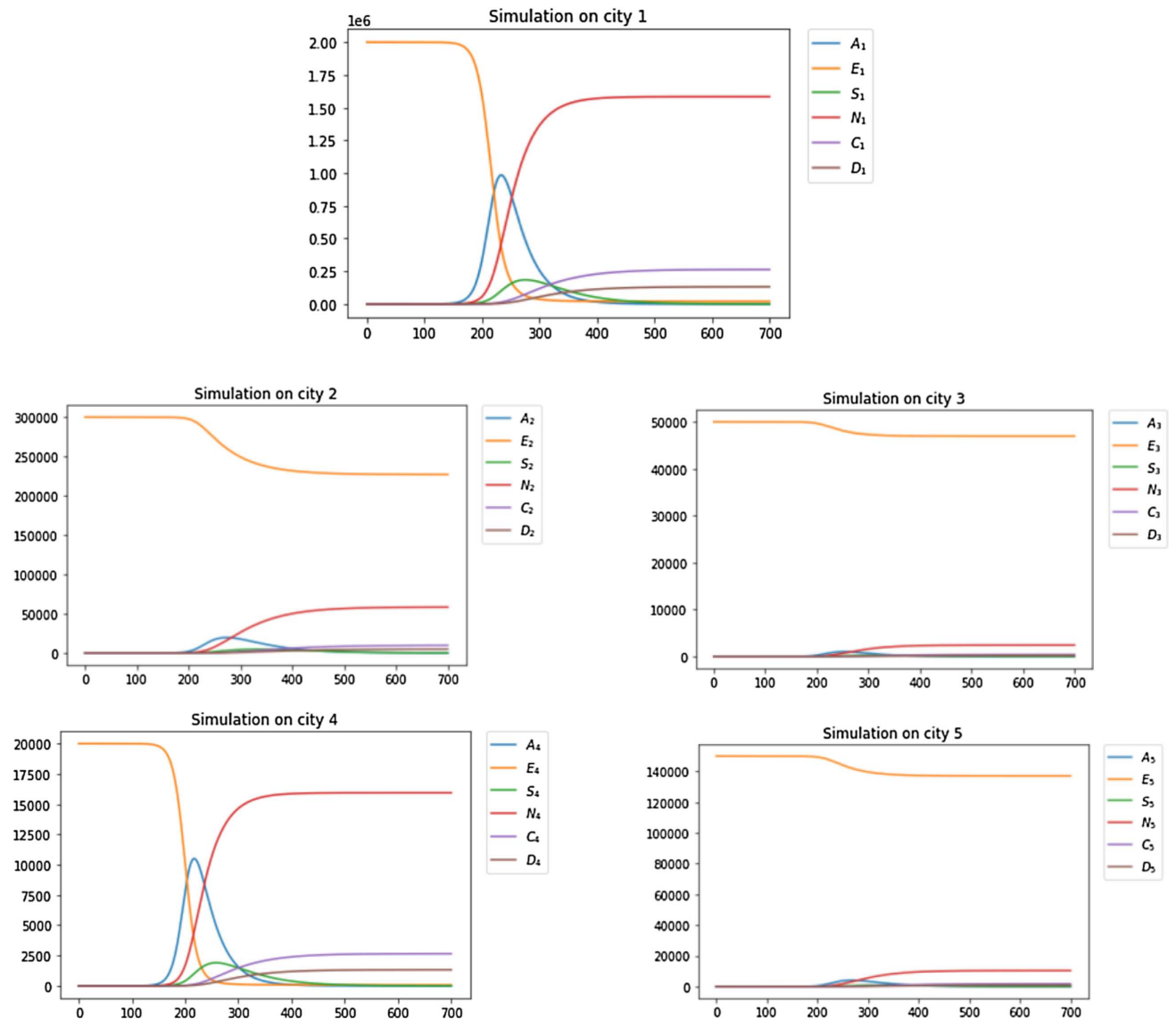

FiguRE 5. Example of regional propagation of a pandemic (no social isolation policy).

a peak and then declines abruptly, the active part of the pandemic taking more than two hundred days. It appears also that the development of the pandemic in the cities 2, 3 and 5 is delayed with respect of the one of the cities 1 and 4 which are the initial sources of infection. Also, by the end of the pandemic, practically all the population of cities 1 and 4 has been touched, while in the cities 2, 3 and 5, a large part of the population remains untouched.

A second round of simulation has been performed where the pandemic starts with a unique infected person at city 5, the results are displayed in Figure 5. The objective of this scenario is to observe the propagation of the pandemic from one city to another. Here also, no social isolation policy is implemented. Here it takes about five months to get a noticed health problem, then the declared infection propagates in few days from city 5 to city 1 and then it takes about six weeks to propagate from city 1 to cities 2,3 and 4 . Observe also that in this case the pandemic appears in city 1 with a delay of two months with respect to the case where this city is an initial infected center.

\section{A STATE SPACE REPRESENTATION OF PANDEMia DYNAMiCS}

Since we are interested in controlling the expension of the pandemic, formal control problems can be formulated. Then a global state space representation has to be built to ease the use of the technics of Control Theory. 
This state representation is built from the previous model where to each city is attached a sub space vector:

$$
\boldsymbol{X}(t)=\left(\boldsymbol{X}_{1}(t)^{\prime}, \boldsymbol{X}_{2}(t)^{\prime}, \ldots, \boldsymbol{X}_{n_{T}}(t)^{\prime}\right)^{\prime}
$$

with

$$
\boldsymbol{X}_{i}(t)=\left(A_{i}(t), S_{i}(t), N_{i}(t), C_{i}(t), D_{i}(t)\right)^{\prime}, \quad i=1 \text { to } n_{T} .
$$

Then the global state representation adopts the following classical affine form of state dimension equal to $4 \cdot n_{T}$ with at most $n_{T} \cdot n_{T}$ control entries:

$$
\dot{\boldsymbol{X}}(t)=\boldsymbol{f}(\boldsymbol{X}(t-\tau))+\boldsymbol{g}(\boldsymbol{X}(t)) \cdot\left[T_{i j}(t)\right]
$$

where $\boldsymbol{f}$ and $\boldsymbol{g}$ is a continuously differentiable vectorial functions where the $E_{i}$ are expressed according to relation (3.9). Since the presence of pure delays in the state equation (6.4) does not allow to apply directly the Minimum Principle approach to the solution of optimal control problems considering it, a first order approximation of delays is performed.

with

$$
\boldsymbol{f}=\operatorname{diag}\left\{\boldsymbol{f}_{1}, \ldots, \boldsymbol{f}_{n_{T}}\right\}
$$

$$
\boldsymbol{f}_{i}\left(\boldsymbol{X}_{i}(t-\boldsymbol{\tau})\right)=\left[\begin{array}{c}
-\rho_{A N i} A_{i}\left(t-\tau_{N i}\right)-\rho_{A S i} A_{i}\left(t-\tau_{S i}\right) \\
\rho_{A S i} A_{i}\left(t-\tau_{S i}\right)-\rho_{S C i} S_{i}\left(t-\tau_{T i}\right)-\rho_{S D i} S_{i}\left(t-\tau_{D i}\right) \\
\rho_{A N i} A_{i}\left(t-\tau_{N i}\right) \\
\rho_{S C i} S_{i}\left(t-\tau_{T i}\right) \\
\rho_{S D i} S_{i}\left(t-\tau_{D i}\right)
\end{array}\right] i=1, \ldots, n_{T}
$$

When adopting the first order approximation of delays we get:

$$
\boldsymbol{f}_{i}\left(\boldsymbol{X}_{i}(t-\boldsymbol{\tau})\right) \approx \boldsymbol{F}_{i} \cdot \boldsymbol{X}_{i}+\boldsymbol{H}_{i} \cdot \dot{\boldsymbol{X}}_{i}
$$

with

$$
F_{i}=\left[\begin{array}{ccccc}
-\left(\rho_{A N i}+\rho_{A S i}\right) & 0 & 0 & 0 & 0 \\
\rho_{A S i} & -\left(\rho_{S C i}+\rho_{S D i}\right) & 0 & 0 & 0 \\
\rho_{A N i} & 0 & 0 & 0 & 0 \\
0 & \rho_{S C i} & 0 & 0 & 0 \\
0 & \rho_{S D i} & 0 & 0 & 0
\end{array}\right]
$$

and

$$
H_{i}=\left[\begin{array}{ccccc}
\rho_{A N i} \tau_{N i}+\rho_{A S i} \tau_{S i} & 0 & 0 & 0 & 0 \\
-\rho_{A S i} \tau_{S i} & \rho_{S C i} \tau_{S i}+\rho_{S D i} \tau_{D i} & 0 & 0 & 0 \\
-\rho_{A N i} \tau_{N i} & 0 & 0 & 0 & 0 \\
0 & -\rho_{S C i} \tau_{D i} & 0 & 0 & 0 \\
0 & -\rho_{S D i} \tau_{D i} & 0 & 0 & 0
\end{array}\right]
$$

Then, if:

$$
\rho_{A N i} \tau_{N i}+\rho_{A S i} \tau_{S i} \neq 1 \text { and } \rho_{S C i} \tau_{S i}+\rho_{S D i} \tau_{D i} \neq 1
$$

the matrix $\mathbf{1}_{i}-\boldsymbol{H}_{i}$ is invertible and the state representation (6.3) is replaced by:

$$
\dot{\boldsymbol{X}}(t)=\boldsymbol{A} \cdot \boldsymbol{X}(t)+\boldsymbol{G}(\boldsymbol{X}(t)) \cdot\left[T_{i j}(t)\right]
$$

with

$$
\boldsymbol{A}=\operatorname{diag}\left\{\boldsymbol{A}_{1}, \ldots, \boldsymbol{A}_{n_{T}}\right\}
$$


where

$$
\boldsymbol{A}_{i}=\left(1_{i}-\boldsymbol{H}_{i}\right)^{-1} \boldsymbol{F}_{i} \text { and } \boldsymbol{G}(\boldsymbol{X}(t))=\operatorname{diag}\left\{\left(1_{i}-\boldsymbol{H}_{i}\right)^{-1} i=1, \ldots, n_{T}\right\} \cdot \boldsymbol{g}(\boldsymbol{X}(t)) .
$$

Observe that this new state representation remains affine with respect to the control matrix $\left[T_{i j}\right]$, i.e. the isolation policy. The input matrix is subject to constraints such as:

$$
T_{i j}^{\min } \leq T_{i j}(t) \leq T_{i j}^{n} \quad i \in\left\{1, \ldots, n_{T}\right\} \quad \text { and } \quad j \in\left\{1, \ldots, n_{T}\right\}
$$

with initial conditions:

$$
\boldsymbol{X}_{i}(0)=\left(A_{i}(0), S_{i}(0), N_{i}(0), C_{i}(0), D_{i}(0)\right)^{\prime} \quad i=1 \text { to } n_{T} .
$$

Here we consider different optimization problems where the adopted performance is estimated over a time period $\left[0, t_{f}\right]$ where $t_{f}$ is the time at which the pandemia can be considered in its final phase before extinction. It is supposed that this time is characterized by the conditions:

$$
\pi_{i i}\left(t_{f}\right) \leq p_{i}^{\max } \quad i=1, \ldots, n_{T}
$$

where $p_{i}^{\max }$ is a maximum threshold level for the probability of infection of an exposed people by an asymptomatic one. To avoid to consider $n_{T} \times n_{T}$ conditions, it is considered that if infection becomes much less probable in each center, this will reflect positively on the cross infection levels between centers. Probability $\pi_{i i}(t)$ goes to zero either when $A_{i}(t)$ goes to zero (there are no more asymtomatic people) or when $E_{i}(t)$ goes to zero (there all the population at city $i$ has been infected). Observe that $A_{i}(t)$ and $N_{i}(t)$ can be estimated through testing of a sample of the population which is not symptomatic $S_{i}(t)$, cured $C_{i}(t)$ or dead $D_{i}(t)$ and then $E_{i}(t)$ can also be estimated according to (3.8). Conditions (6.15) is replaced here by equality:

$$
\begin{gathered}
\left(P_{i}-\left(A_{i}\left(t_{f}\right)+S_{i}\left(t_{f}\right)+N_{i}\left(t_{f}\right)+C_{i}\left(t_{f}\right)+D_{i}\left(t_{f}\right)\right) \cdot A_{i}\left(t_{f}\right)\right. \\
-p_{i}^{\max }\left(P_{i}-\left(S_{i}\left(t_{f}\right)+D_{i}\left(t_{f}\right)\right)\right)^{2}=0 \quad i=1, \ldots, n_{T}
\end{gathered}
$$

or

$$
\varphi_{i}\left(\boldsymbol{X}_{i}\left(t_{f}\right)\right)=0 \quad i=1, \ldots, n_{T}
$$

\section{Pandemic Control in minimum time}

A possible objective for pandemia control is to minimize the duration of the pandemic. This objective is related with economic and social considerations where it is of interest to return to normal activity levels as soon as possible. Let this problem be Problem 1 which can be formulated as:

$$
\min _{\left[T_{i j}\right]} t_{f}
$$

under the constraints (6.10), (6.13), (6.14) and (6.17).

To Problem 1 is associated the following Hamiltonian:

$$
H\left(\boldsymbol{X}(t),\left[T_{i j}(t)\right], \boldsymbol{\lambda}(t)\right)=\boldsymbol{\lambda}(t)^{\prime} \cdot\left(\boldsymbol{A} \cdot \boldsymbol{X}(t)+\boldsymbol{G}(\boldsymbol{X}(t)) \cdot\left[T_{i j}(t)\right]\right)
$$

where the vector of adjoint variables is such as:

$$
\boldsymbol{\lambda}(t)=\left(\boldsymbol{\lambda}_{1}(t)^{\prime}, \boldsymbol{\lambda}_{2}(t)^{\prime}, \ldots, \boldsymbol{\lambda}_{n_{T}}(t)^{\prime}\right)^{\prime}
$$

with

$$
\boldsymbol{\lambda}_{i}(t)=\left(\lambda_{A_{i}}(t), \lambda_{S_{i}}(t), \lambda_{N_{i}}(t), \lambda_{C_{i}}(t), \lambda_{D_{i}}(t)\right)^{\prime}, \quad i=1 \text { to } n_{T} .
$$


This Hamiltonian can also be rewritten as:

$$
\begin{aligned}
H\left(\boldsymbol{X}(t),\left[T_{i j}(t)\right], \boldsymbol{\lambda}(t)\right)= & \boldsymbol{\lambda}(t)^{\prime} \cdot f(\boldsymbol{X}(t))+\sum_{i=1}^{n_{T}} \alpha_{i} \cdot \lambda_{A_{i}}(t) \cdot \beta_{i i} \cdot \pi_{i i}(t) \cdot T_{i i}(t) \\
& +\sum_{i=1}^{n_{T}} \sum_{j=1, j \neq i}^{n_{T}} \alpha_{i}\left(\lambda_{A_{i}}(t) \cdot \beta_{j i} \cdot \pi_{i j}(t)+\lambda_{A_{j}}(t) \cdot \beta_{i j} \cdot \pi_{j i}(t)\right) \cdot T_{i j}(t)
\end{aligned}
$$

with

$$
\alpha_{i}=1 /\left(1-\left(\rho_{A N i} \tau_{N i}+\rho_{A S i} \tau_{S i}\right)\right) .
$$

Necessary optimality conditions can be written here according to the Minimum Principle of Pontryagine $[6,9]$ :

$$
\begin{aligned}
\dot{\boldsymbol{X}}^{*}(t) & =\boldsymbol{A} \cdot \boldsymbol{X}^{*}(t)+\boldsymbol{g}\left(\boldsymbol{X}^{*}(t)\right) \cdot\left[T_{i j}^{*}(t)\right] \quad \text { with } \quad \boldsymbol{X}^{*}(t)=\boldsymbol{X}(0) \\
\dot{\boldsymbol{\lambda}} & =-\frac{\partial H}{\partial \boldsymbol{X}}\left(\boldsymbol{X}^{*}(t),\left[T_{i j}(t)\right], \boldsymbol{\lambda}^{*}(t)\right) \\
{\left[T_{i j}^{*}(t)\right] } & =\arg \left\{\min _{\left[T_{i j}\right]} H\left(\boldsymbol{X}^{*}(t),\left[T_{i j}(t)\right], \boldsymbol{\lambda}^{*}(t)\right)\right\}
\end{aligned}
$$

with the control contraints (6.13), with the terminal conditions (6.17) and the transversality conditions:

$$
\begin{array}{r}
H\left(\boldsymbol{X}\left(t_{f}\right),\left[T_{i j}\left(t_{f}\right)\right], \boldsymbol{\lambda}\left(t_{f}\right)\right)+1=0 \\
\boldsymbol{\lambda}\left(t_{f}\right)-\boldsymbol{v}^{\prime} \cdot \partial \boldsymbol{\varphi}\left(\boldsymbol{X}\left(t_{f}\right)\right) / \partial \boldsymbol{X}\left(t_{f}\right)=0
\end{array}
$$

where is a vector of Lagrange multipliers of dimension $5 \cdot n_{T}$. Then for $i=1$ to $n_{T}$ :

$$
\begin{aligned}
\partial \boldsymbol{\varphi}_{\boldsymbol{i}}(\boldsymbol{X}(t)) / \partial \boldsymbol{A}_{\boldsymbol{i}}(t) & =E_{i}(t)-A_{i}(t) \\
\partial \boldsymbol{\varphi}_{\boldsymbol{i}}(\boldsymbol{X}(t)) / \partial \boldsymbol{S}_{\boldsymbol{i}}(t) & =-A_{i}(t)+2 \cdot p_{i}^{\max }\left(P_{i}-S_{i}(t)\right) \\
\partial \boldsymbol{\varphi}_{\boldsymbol{i}}(\boldsymbol{X}(t)) / \partial \boldsymbol{N}_{\boldsymbol{i}}(t) & =\partial \boldsymbol{\varphi}_{\boldsymbol{i}}(\boldsymbol{X}(t)) / \partial \boldsymbol{C}_{\boldsymbol{i}}(t)=-A_{i}(t) \\
\partial \boldsymbol{\varphi}_{\boldsymbol{i}}(\boldsymbol{X}(t)) / \partial \boldsymbol{D}_{\boldsymbol{i}}(t) & =-A_{i}(t)+2 \cdot p_{i}^{\max }\left(P_{i}-D_{i}(t)\right) .
\end{aligned}
$$

Considering the affine form of the Hamiltonian with respect to $\left[T_{i j}\right]$ and restrictions (6.13) it appears that the solution of problem (7.1) is when the $\alpha_{i} i=1$ to $n_{T}$ are positive, such that:

$$
\begin{aligned}
\text { if } \lambda_{A_{i}}^{*}(t) & >0 \text { then } T_{i i}^{*}(t)=T_{i i}^{\min } \quad i=1, \ldots, n_{T} \\
\text { if } \lambda_{A_{i}}^{*}(t)<0 \text { then } T_{i i}^{*}(t) & =T_{i i}^{n} \quad i=1, \ldots, n_{T} \\
\text { if } \lambda_{A_{i}}(t) \cdot \beta_{j i} \cdot \pi_{i j}(t)+\lambda_{A_{j}}(t) \cdot \beta_{i j} \cdot \pi_{j i}(t) & >0 \text { then } T_{i j}^{*}(t)=T_{j i}^{*}(t)=T_{i j}^{\min } \quad i \neq j \\
\text { if } \lambda_{A_{i}}(t) \cdot \beta_{j i} \cdot \pi_{i j}(t)+\lambda_{A_{j}}(t) \cdot \beta_{i j} \cdot \pi_{j i}(t)<0 \text { then } T_{i j}^{*}(t) & =T_{j i}^{*}(t)=T_{i j}^{n} \quad i \neq j .
\end{aligned}
$$

From relations (7.12) it appears that the optimal solution is of the bang-bang type. So in theory, every time $\lambda_{A_{i}(t)}$ changes its sign, there should be a shift of $T_{i i}$ from an extreme value to the other. Solving numerically Problem 1 to obtain the optimal trajectory of $\lambda_{A_{i}}$ is a difficult task. However since here what is sought is a policy which leads to the minimum duration for the pandemia, every extreme shift will delay the evolution of the pandemia to the final equilibrium since the previous trend of increasing or decreasing the stocks of asymptomatic and symptomatic people will be reversed. The same reasoning can be applied to the connections between the different towns and more, in that case the adoptions of different policies for the interactions between different towns will result also in increased delays towards the final equilibrium. So, two candidate solutions remain:

- Adopt during the period $\left[0, t_{f}\right]$ the solution:

$$
T_{i j}=T_{i j}^{\min }, \quad i=1 \text { to } n_{T}, \quad j=1 \text { to } n_{T} .
$$




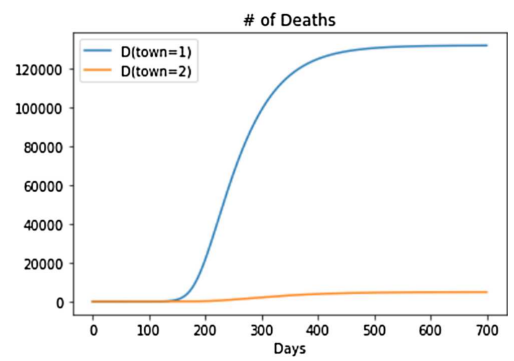

FiguRE 6. Number of deaths with no social isolation.

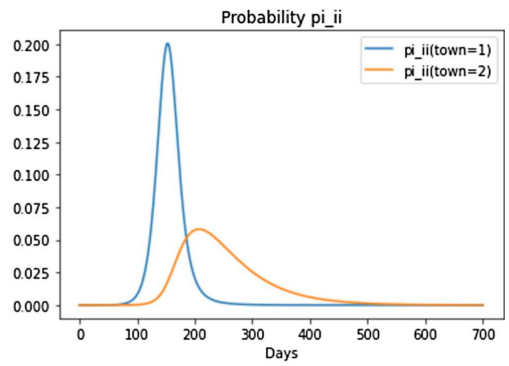

FiguRE 7. Probabilities of interaction during pandemic with no social isolation.

- Adopt during period $\left[0, t_{f}\right]$ the solution:

$$
T_{i j}=T_{i j}^{n}, \quad i=1 \text { to } n_{T}, \quad j=1 \text { to } n_{T} .
$$

In the first case interaction between people will be maintained at a minimum level so that people will get infected very slowly and that will make probability $\pi_{i i}(t)$ go to zero with $A_{i}(t)$, then people will recover with a reduced death toll. In the second case, the idea is to infect quickly as much people as possible so that the number of exposed people in each town will be drastically reduced (see relation (3.3)) with $E_{i}(t)$ going to zero and leading rapidly to final equilibrium, however this may imply a higher death toll since more people will become symptomatic and eventually, die. Then, the solution of the minimum time pandemic control is given by (7.13b): no isolation at all. This corresponds to the situation displayed by Figure 3. Figure 6 displays the detailed evolution of the number of deaths in cities 1 and 2, leading respectively to the final scores of 130000 and 3000. In Figure 7 is displayed the evolution of the probabilities of interaction $\pi_{11}$ and $\pi_{22}$, displaying the effective period of the pandemic in cities 1 and 2. In the case of city 1 , the minimum duration of the pandemic is about five months, while for city 2 the minimum duration of the pandemic is about ten months.

Figure 9 give more detailed views of the pandemic history of cities 1 and 2. It appears that with this policy, the death toll is now of 70000 people instead of 130000 , however, long duration isolation is not acceptable from economic and social points of view.

\section{Pandemia CONTROL FOR Minimum DeAth TOLL}

Another important objective for pandemia control is to minimize the death toll during the whole pandemia. This objective is related not only with humanitarian considerations but also with economic considerations since in that case, return to normal activity levels may be performed more efficiently. Let this problem be Problem 2 

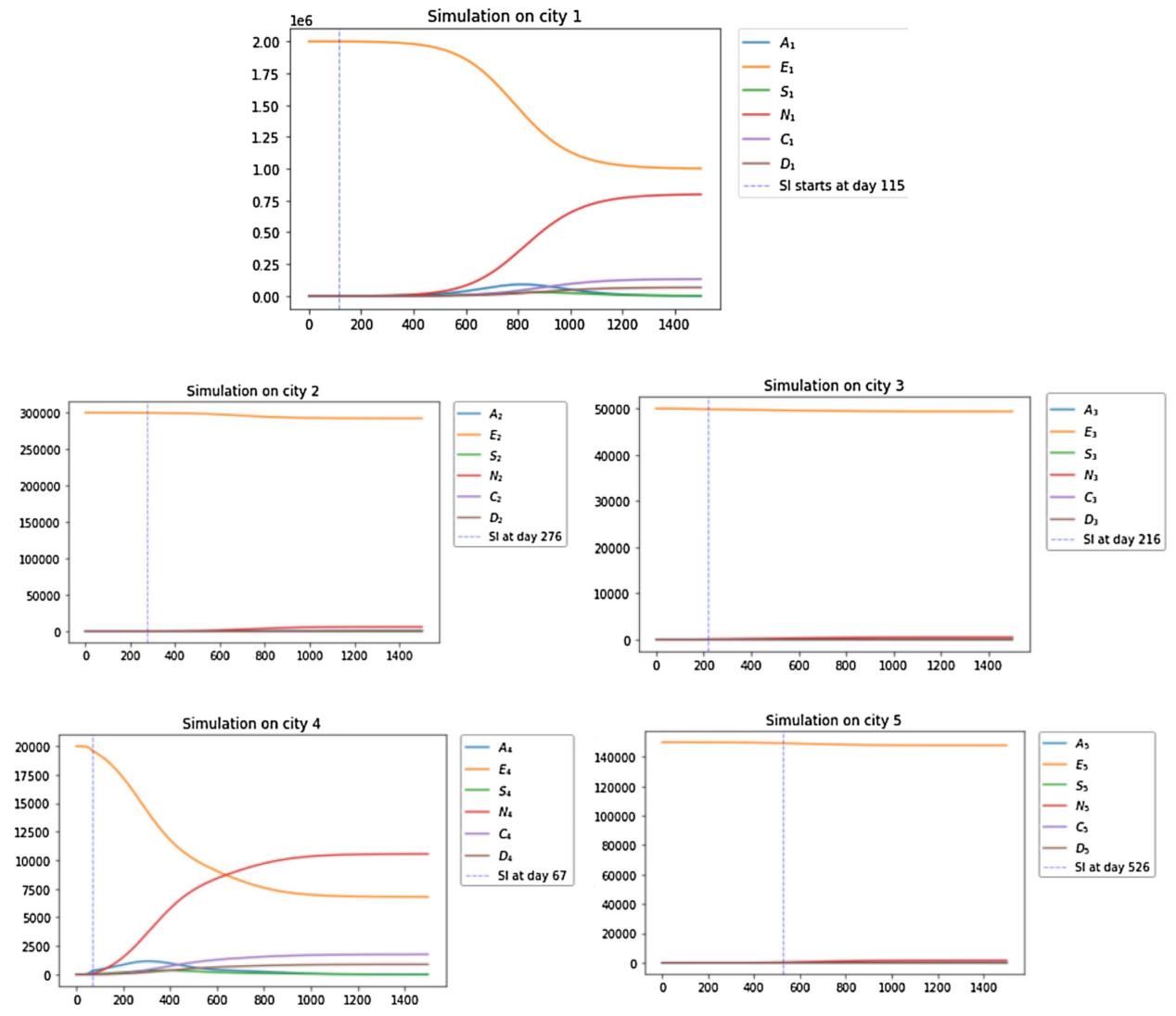

Figure 8. Applying a long isolation policy.

which can be formulated as:

$$
\min _{\left[T_{i j}\right]} \psi\left(\boldsymbol{X}\left(t_{f}\right)\right) \quad \text { with } \quad \psi\left(\boldsymbol{X}\left(t_{f}\right)\right)=\sum_{i=1}^{n_{T}} D_{i}\left(t_{f}\right)
$$

under the constraints $(6.10),(6.13),(6.14)$ and (6.17). Here also, the terminal time is chosen according to relation (6.16). The Hamiltonian associated to Problem 2 is the same as for Problem 1 (relations (7.2)-(7.4)). Then the necessary conditions (7.6), (7.7), (7.8) with (6.13) and (6.17) remain valid. Now the transversality conditions are written:

$$
\begin{aligned}
H\left(\boldsymbol{X}\left(t_{f}\right),\left[T_{i j}\left(t_{f}\right)\right], \boldsymbol{\lambda}\left(t_{f}\right)\right) & =0 \\
\boldsymbol{\lambda}\left(t_{f}\right)-\partial\left(\boldsymbol{X}\left(t_{f}\right)\right) / \partial \boldsymbol{X}\left(t_{f}\right)-v \cdot \partial \boldsymbol{\varphi}\left(\boldsymbol{X}\left(t_{f}\right)\right) / \partial \boldsymbol{X}\left(t_{f}\right) & =0 .
\end{aligned}
$$

Here again, the numerical resolution of these necessary optimality conditions is difficult and their further analysis can lead to the solution: considering condition (7.8) and the affine form of the Hamiltonian, it is again expected that the optimal solution is from the bang-bang type and following a similar reasoning than in the previous case, it can be expected that there will be no shift during the control horizon. Considering the fact that the transversality conditions (8.2) and (8.3) are different from the previous ones (7.9) and (7.10), the history of the adjoint variables will be different and a diffrent solution can be expected. In fact, considering the analysis developed in the previous section, the only possible solution of the min death toll problem is given by expression (7.13a). 

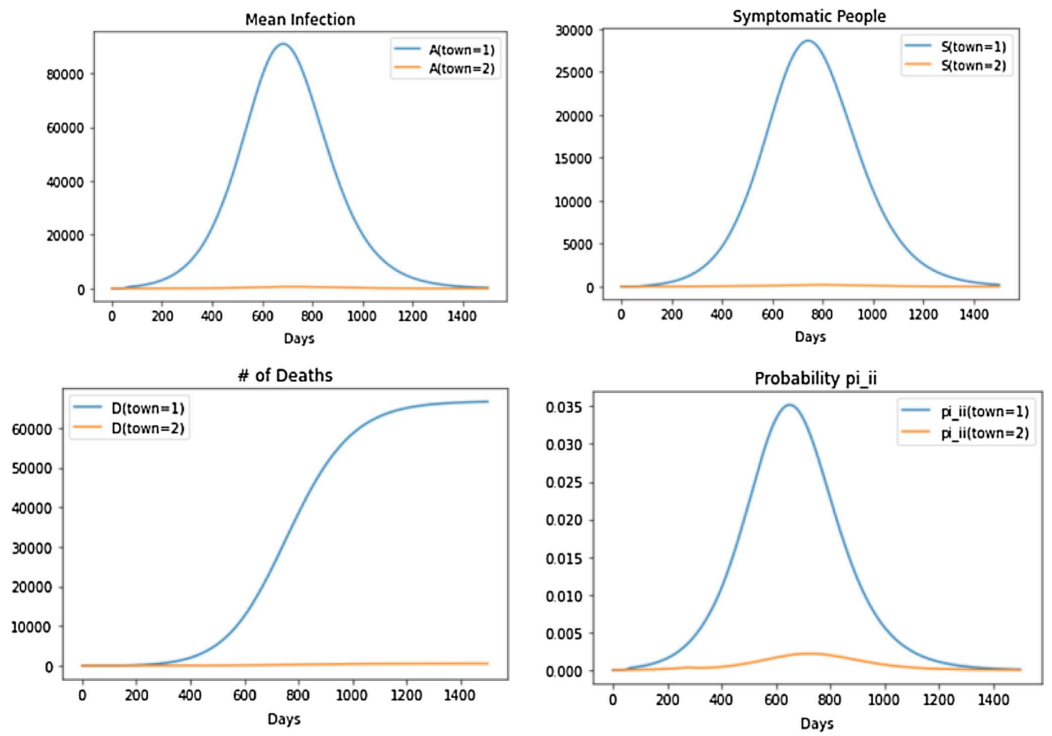

FiguRE 9. Performance of permanent isolation policy on cities 1 and 2.

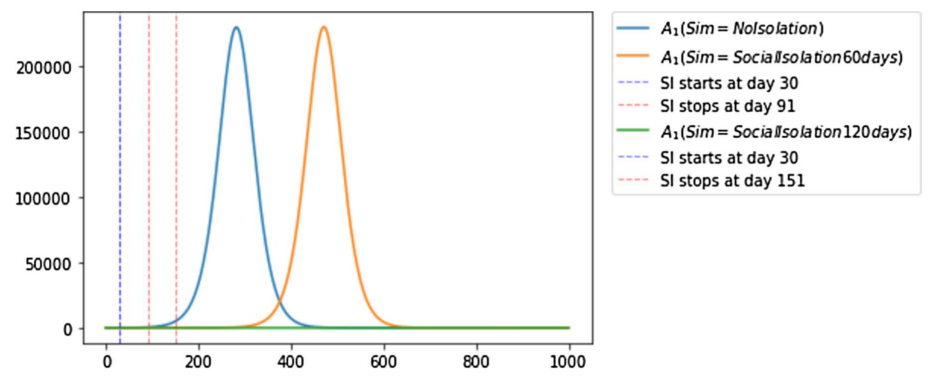

FIGURE 10. Effects on city 1 of different isolation policies.

Simulations have been performed where the isolation policy has been applied, it has been considered that during isolation, the number of interactions between people is divided by 3 . Two points have to be solved: when to start the isolation policy and when to put an end to isolation. For the first point, it has been considered that isolation starts at a city when a minimum number of symptomatic people is reached. For the second point it was considered that the fulfilment of condition (6.15) with a minimum value of $10^{-9}$ will put an end to the pandemic. A first round of simulations has been performed with symptomatic threshold values of respectively $50,10,5,5$ and 5 for the cities $1-5$. The simulation results are displayed in Figure 8 where it appears that according to the chosen end criteria, the pandemic could last for 1500 days.

Thus, it was decided to apply the isolation policy only for a limited period of time starting at the detection of symptomatic cases when their numbers reach the above threshold values. Here for brievity, only the resulting asymptomatic history of city 1 is displayed in Figure 10 where three cases are considered: no isolation, isolation policy applied for only a month and isolation policy applied for two months. It appears that the isolation policy of one month achieves only to postpone the advent of the critical phase of the pandemic for two hundred days, while with an isolation of two months the infection which is not complectly finished adopts an endemic behaviour, giving time to improve medication or to find an effective vaccine. Also, the death toll, even when waiting for the complete ending of the pandemic remains much smaller than with a minimum time policy. 


\section{Conclusion}

In this study an extension of the SIR models has been developed to represent the spatial dynamics of a pandemic and to allow the analyzis and comparison of the performances of different social isolation policies since their are, according to WHO the ultimate tools when no effective treatment is at hand or when medical resources are under stress. This theoretical model which considers the main components of the dynamics of pandemic has not been calibrated using field data and is largely improvable. Nevertheless, it has already shown its compatibility with Optimal Control Theory and has displayed capabilities to help to understand and fix some key factors for a successful fight against pandemic .

\section{REFERENCES}

[1] M. Abdullah, C. Cooper and M. Draief, Viral processes by random walks on random regular graphs. Ann. Appl. Probab. 25 (2015) 477-522.

[2] G.G. Alcaraz and C. Vargas-De-Leon, Modeling control strategies for influenza H1N1 epidemics: SIR models. Rev. Mex. Fis. $S 58$ (2012) 37-43.

[3] R. Anguelov, J. Banasiak, C. Bright and R. Ouifki, The big unknown: the asymptomatic spread of COVID-19. J. Biomath. 9 (2020) 2005103.

[4] U.S. Basak1, B.K. Datta and P.K. Ghose, Mathematical analysis of an HIV/AIDS epidemic model. Am. J. Math. Stat. 5 (2015) 253-258.

[5] F. Brauer and C. Castillo-Chavez, Mathematical Models in Population Biology and Epidemiology. Springer (2012).

[6] R.M. Faye and F. Mora-Camino, La Commande Optimale, L'Harmattan, Paris (2017).

[7] A. Flahault, S. Deguen and A.-J. Valleron, A mathematical model for the European spread of influenza. Eur. J. Epidemiol. 10 (1994) 471-474.

[8] H.W. Hethcote, The mathematics of infectious diseases. SIAM Rev. 42 (2000) 599-653.

[9] A.D. Lewis, The maximum principle of pontryaginin in optimal control. Available from: https://www.ime.usp.br/ tonelli/ pub/maximum-principle.pdf (2006).

[10] Z. Liu, P. Magal, O. Seydi and G. Webb, Predicting the cumulative number of cases for the COVID-19 epidemic in China from early data. Preprint medRXiv DOI: 10.1101/2020.03.11.20034314 (2020).

[11] G.C.E. Mbah, D. Omale, B. O. Adejo, A SIR epidemic model for HIV/AIDS infection. Int. J. Sci. Eng. Res. 5 (2014) 479-484.

[12] B. Mbaye Ndiaye, L.Tendeng and D. Seck, Analysis of the COVID-19 pandemic by SIR model and machine learning technics for forecasting. Preprint arXiv: 2004.01574v1 [q-Bio-PE] (2020).

[13] T.W. Ng, G. Turinici and A. Danchin, A double epidemic model for the SARS propagation. BMC Infectious Diseases 3 (2003) 19.

[14] B.S. Pujari and S. Shekatka, Multi-city modeling of epidemics using spatial networks: application to 2019-nCov (COVID-19) coronavirus in India. Preprint medRxiv DOI: 10.1101/2020.03.13.20035386 (2020).

[15] WHO, Available from: https://www.who.int/csr/disease (2010).

[16] O. Zakary, M. Rachik and I. Elmouki, Multi-regions discrete SIR epidemic model: an optimal control approach. Int. J. Dyn. Control 5 (2017) 917-930. 